\title{
Bone Density and Exercise
}

\author{
Christina L Silva* \\ Associate Professor, Department of Nursing, Slippery Rock University, USA
}

*Corresponding author: Christina L Silva, Associate Professor, Department of Nursing, Slippery Rock University, USA.

\author{
Received Date: August 15, 2019 \\ Published Date: August 23, 2019
}

\section{Introduction}

In the world of nursing and medicine, many diseases and physical or mental impairments are treated with pharmaceuticals or medication. For some diseases such as type 1 diabetes, a pharmaceutical or medication, insulin, is the proper treatment and one that is absolutely necessary. Not all conditions fall into the category of required medications; a couple of these conditions would be osteoporosis and osteopenia. The treatment guidelines call for medications based on $\mathrm{t}$-scores and fracture risk Tuck $\mathrm{S}$, et al. [1], even if the medication has bad or life-threatening side effects and even if there are other potential treatments that can be effective.

In a search of the literature, osteoporosis treatment is built around the use of pharmaceutical treatment regimens [1,2,3]. The treatment regimens are based on dual energy $\mathrm{x}$-ray absorptiometry (DXA) t-scores and history of patient fractures. Examples of the treatment guidelines include bio phosphonates for $\mathrm{T}$ scores $<-2.5$, denosumab if patient intolerant of bio phosphonates and T-score $<-2.5$ or history of fracture, consider teriparatide as second line treatment if patient has issues with oral bisphosphonates and $\mathrm{T}$ score $<-2.5$ or history of fragility fracture [1]. In all of the studies reviewed, weight bearing exercise was not mentioned. Hind K \& Roberts C [4] concluded that we have an osteoporosis epidemic today due to increased amounts of sedentary activities. In children, more are playing video games instead of outside running and jumping. In older adults, retirement has become filled with sitting and sedentary activities. Pharmaceuticals can only do so much.

When using medications such as denosumab, the patient is at a higher risk of fracture after the discontinuation of the medication especially if the patient had been on bio phosphonates previously [5]. With medications abounding, it can be easy to prescribe a magic pill, but a better solution might be more physical activity. Even though each medication has a potential side effect, medication is the first treatment modality offered to patients. Another treatment modality that could be offered is weight bearing exercise. Lack of weight bearing exercise is listed as a risk factor for osteoporosis, Faust V [6] but little is found about its potential as a treatment modality. As an osteoporotic patient, the author, has experienced this scenario for years. At initial diagnosis of osteopenia, weight bearing exercise was mentioned in passing yet medications were ordered and discussed as the way to approach this potentially debilitating condition. The idea of non-compliance with the medication was not discussed nor were alternatives to medication. Once the diagnosis progressed to osteoporosis and post bone fracture, more advanced medications were ordered. These medications are more invasive and require injection of denosumab either every 6 months or the use of teriparatide daily [1]. Even with the need for more invasive types of medication, the use of weight bearing activity remained an unspoken alternative. The following is a case study of the author's medical history and a call for more holistic and alternative methods to be used in treatment of osteoporosis [7].

\section{Case Study}

Thepatientis a slender,Caucasianfemale who had ahysterectomy and bilateral oophorectomy at the age of 27 and comorbidities of gastroesophageal reflux disease and endometriosis. By the age of 35 , she had the diagnosis of osteopenia and was ordered the first line treatment of bisphosphonates. Unfortunately, she had a comorbidity of gastroesophageal reflux and was unable to take the first line treatment. The patient was prescribed denosumab after this but with preauthorization requirements, it took a year and a half to get the medication approved. During this period, exercise was mentioned but it was not prescribed as a treatment. In 2010, the patient had suffered a radial fracture from a fall and in 2015 she had a fifth metatarsal fracture from landing on her foot wrong. The radial fracture required a cast, but the fifth metatarsal fracture required an open reduction internal fixation with 2 plates and 6 pins. It was after the 2015 injury that denosumab was discontinued and teriparatide was discussed and initiated. Teriparatide was used by patient for about 19 months. 
After it was discontinued, the patient did not have any medication prescribed. In August 2018, the patient began a weightlifting regimen per her own want for a healthier body. As per guidelines, a DXA was performed every two years starting in 2015. For associated t-scores, treatment modality, and major side effects, refer to Table 1.

Table 1: Patient treatments and subsequent results.

\begin{tabular}{|c|c|c|c|c|}
\hline Year & Treatment & Side Effects & T-score Before Treatment & T-score After Treatment \\
\hline 2015 & Forteo-sq injections daily & $\begin{array}{c}\text { Risk of CA if used more than 2 years, } \\
\text { joint pain }\end{array}$ & $-2.8(2015)$ & $-2.6(2017)$ \\
\hline 2018 & $\begin{array}{c}\text { Weight bearing exercise 4 times a } \\
\text { week (patient imposed treatment) }\end{array}$ & $\begin{array}{c}\text { Build muscle mass, increased energy, } \\
\text { increased muscle strength }\end{array}$ & $-2.6(2017)$ & $-2.2(2019)$ \\
\hline
\end{tabular}

\section{Discussion}

For a young patient who had previously only been prescribed medication as a treatment with minimal increase in t-score, the double T-score increase after only nine months of weight bearing exercise was astonishing. This leads to questioning why weight bearing exercise had not been a prescribed treatment? Why is the first line a pharmaceutical agent even when the patient is young? Why are insurance companies not paying for a gym membership or weight machines or other devices to allow for weight bearing exercise to be part of a treatment? Not all patients can afford to go to the gym and not all patients diagnosed with osteopenia or osteoporosis are of Medicare age that would allow for a covered gym program, the silver sneakers program (https://www.medicareproviders.net/plans/fitness?Creative). Medicare supplemental plans are realizing the benefits of seniors having to access to a gym. Will other health insurance companies see a need for non medicated consumers to have access to a gym at a reasonable cost? Instead of making medication a first line treatment for all patients, the protocol should include ordering weight bearing exercise for those who are capable of performing it. The side effects are all positive- weight loss, building of muscle mass, healthy self-image, and of course an increase in bone density. The results could be beneficial to so many other disease processes too. For medical professionals that believe in alternative solutions, could this be considered? Could insurance
As is shown in Table 1 , the patient had positive outcomes with both the teriparatide and weightlifting. The teriparatide was a 19month regimen and the weightlifting was 9 months. The side effects of the teriparatide include bone cancer and joint pain. The side effects of the weightlifting include building of muscle mass, increased energy, and muscle strength. Both have a positive effect on bone density. companies be lobbied to add this type of treatment? The positives of this type of treatment far outweigh the difficulty in finding a way to get it covered.

\section{Acknowledgement}

None.

\section{Conflict of Interest}

No conflict of interest.

\section{References}

1. Tuck S, Little EA, Aspray TJ (2018) Implications of guidelines for osteoporosis and its treatment. Age Aging 47(3): 334-339.

2. Liang J, Chen C, Liu H, Liu X, Li Z, et al (2018) Gossypol promotes bone formation in ovariectomy-induced osteoporosis through regulating cell apoptosis. BioMed Research International 2018: 1-9.

3. (2019) Osteoporosis treatment: What we don't know. Pharmacy\& Therapeutics 44(7): 430-435.

4. Hind K, Roberts C (2018) Why hunter-gatherers had stronger bones and how we can get them. The Epoch Times 44(7): 430.

5. Tripto Shkolnik L, Rouach V, Marcus Y, Rotman Pikielny P, Benbassat C, et al. (2018) Vertebral fractures following denusumab discontinuation in patients with prolonged exposure to bisphosphonates. Calcif Tissue Int 103(1): 44-49.

6. Faust V (2019) Aging and the risk for hip fractures. Philadelphia, USA, pp. 8A-9A.

7. Gorini M (2019) Newer isn't better when it comes to osteo treatment. The Women's Health Activist 44(3): 3. 